\title{
Predictive value of anti-CarP and anti-PAD3 antibodies alone or in combination with RF and ACPA on the severity of rheumatoid arthritis
}

Lamacchia, Celine ; Courvoisier, Delphine S ; Jarlborg, Matthias ; Bas, Sylvette ; Roux-Lombard, Pascale ; Möller, Burkhard ; Ciurea, Adrian ; Finckh, Axel ; Bentow, Chelsea ; Martinez-Prat, Laura ; Mahler, Michael ; Gabay, Cem ; Nissen, Michael J

\begin{abstract}
OBJECTIVES To analyse the predictive value of anti-carbamylated protein (anti-CarP) and anti-peptidyl-arginine deiminase type-3 (anti-PAD3) antibodies, alone or in combination with rheumatoid factors (RFs) and anti-citrullinated protein antibodies (ACPA), to identify patients at high risk of developing severe rheumatoid arthritis (RA) outcomes. METHODS Patients within the « Swiss Clinical Quality Management » registry with a biobank sample were tested for RFs, ACPA, anti-CarP, and anti-PAD3 antibodies. We examined the association of each autoantibody with DAS28, HAQ and radiographic damage (Ratingen) at baseline and longitudinally. RESULTS Analyses included 851 established RA patients and 516 disease controls [axial spondyloarthritis $($ axSpA $=320)$ and psoriatic arthritis $\left(\mathrm{PsA}_{\mathrm{s}}\right.$ $=196)$ ]. Anti-CarP and anti-PAD3 antibodies were respectively present in $22.4 \%$ and $10.7 \%$ of the whole RA population, and in $13.2 \%$ and $3.8 \%$ of the RF and ACPA double seronegative patients. At baseline, RA patients with anti-PAD3 had higher DAS28 (4.2 vs 3.7; p = 0.005) and significantly more radiographic damage (14.9 vs 8.8; $\mathrm{p}=0.02$ ) than anti-PAD3 negative patients. In ACPA negative subgroup, baseline Ratingen scores were significantly higher in anti-PAD3 positive patients $(\mathrm{p}=0.01)$. The combination of anti-PAD3, RF IgM, and ACPA was associated with significantly higher baseline radiographic scores than the double seropositive group $(\mathrm{p}=0.04)$. The presence of any two of the previous autoantibodies was associated with significantly greater radiographic progression over 10 years than if all were absent $(\mathrm{p}$ $=0.02)$. There were no differences on RA outcome measures with regards to anti-CarP. CONCLUSIONS Anti-PAD3 antibodies are associated with higher disease activity and joint damage scores in RA patients.
\end{abstract}

DOI: https://doi.org/10.1093/rheumatology/keab050

Posted at the Zurich Open Repository and Archive, University of Zurich

ZORA URL: https://doi.org/10.5167/uzh-198733

Journal Article

Accepted Version

Originally published at:

Lamacchia, Celine; Courvoisier, Delphine S; Jarlborg, Matthias; Bas, Sylvette; Roux-Lombard, Pascale; Möller, Burkhard; Ciurea, Adrian; Finckh, Axel; Bentow, Chelsea; Martinez-Prat, Laura; Mahler, Michael; Gabay, Cem; Nissen, Michael J (2021). Predictive value of anti-CarP and anti-PAD3 antibodies alone or in combination with RF and ACPA on the severity of rheumatoid arthritis. Rheumatology, 60(10):4598-4608.

DOI: https://doi.org/10.1093/rheumatology/keab050 
Predictive value of anti-CarP and anti-PAD3 antibodies alone or in combination with RF and ACPA on the severity of rheumatoid arthritis.

Celine Lamacchia ${ }^{1}$, Delphine S. Courvoisier ${ }^{1}$,Matthias Jarlborg ${ }^{1}$, Sylvette Bas ${ }^{1}$, Pascale Roux-Lombard ${ }^{2}$, Burkhard Möller ${ }^{3}$, Adrian Ciurea ${ }^{4}$, Axel Finckh ${ }^{1}$, Chelsea Bentow ${ }^{5}$, Laura Martinez-Prat ${ }^{6}$, Michael Mahler ${ }^{5}$, Cem Gabay ${ }^{1}$, Michael J. Nissen ${ }^{1}$ on behalf of the SCQM Rheumatologists

${ }^{1}$ Department of Rheumatology, Geneva University Hospital, Geneva, Switzerland

${ }^{2}$ Department of Immunology and Allergy, Geneva University Hospital, Geneva, Switzerland

${ }^{3}$ Department of Rheumatology, Immunology and Allergy, Bern University Hospital, Bern, Switzerland

${ }^{4}$ Department of Rheumatology, Zurich University Hospital, Zurich, Switzerland

${ }^{5}$ Research and Development, Inova diagnostics, San Diego, CA, USA

${ }^{6}$ Research and Development, Inova diagnostics, Barcelona, Spain

\section{Corresponding author:}

Michael J. Nissen,

Department of Rheumatology, University Hospital of Geneva

26 Avenue Beau-Sejour,

1211 Geneva 14, Switzerland

E-mail: michael.j.nissen@hcuge.ch

\section{Manuscript content:}

Abstract length: 247 words

Article length: 3725 words

References: 50

Tables: 3; Figures: 3

Supplementary files: supplementary methods, 4 tables and 2 figures.

Keywords: RA, radiographs, ACPA, RF, anti-PAD3, anti-CarP 


\section{ABSTRACT}

Objectives: To analyse the predictive value of anti-carbamylated protein (anti-CarP) and antipeptidyl-arginine deiminase type-3 (anti-PAD3) antibodies, alone or in combination with rheumatoid factors (RFs) and anti-citrullinated protein antibodies (ACPA), to identify patients at high risk of developing severe rheumatoid arthritis (RA) outcomes.

Methods: Patients within the « Swiss Clinical Quality Management » registry with a biobank sample were tested for RFs, ACPA, anti-CarP, and anti-PAD3 antibodies. We examined the association of each autoantibody with DAS28, HAQ and radiographic damage (Ratingen) at baseline and longitudinally.

Results: Analyses included 851 established RA patients and 516 disease controls [axial spondyloarthritis $(\operatorname{axSpA}=320)$ and psoriatic arthritis $(\mathrm{PsA}=196)]$. Anti-CarP and anti-PAD3 antibodies were respectively present in $22.4 \%$ and $10.7 \%$ of the whole RA population, and in $13.2 \%$ and $3.8 \%$ of the RF and ACPA double seronegative patients. At baseline, RA patients with anti-PAD3 had higher DAS28 (4.2 vs. 3.7; $p=0.005)$ and significantly more radiographic damage (14.9 vs. $8.8 ; p=0.02)$ than anti-PAD3 negative patients. In ACPA negative subgroup, baseline Ratingen scores were significantly higher in anti-PAD3 positive patients $(p=0.01)$. The combination of anti-PAD3, RF IgM, and ACPA was associated with significantly higher baseline radiographic scores than the double seropositive group $(p=0.04)$. The presence of any two of the previous autoantibodies was associated with significantly greater radiographic progression over 10 years than if all were absent $(p=0.02)$. There were no differences on RA outcome measures with regards to anti-CarP.

Conclusions: Anti-PAD3 antibodies are associated with higher disease activity and joint damage scores in RA patients.

\section{Key messages}

1. Anti-PAD3 positivity is associated with higher disease activity and joint damage scores, including the ACPA-negative subgroup.

2. Anti-PAD3 and anti-CarP do not predict greater radiographic progression over 10 years in RA patients.

3. Multiple autoantibodies, including RFs, ACPA and anti-PAD3, may predict the severity of radiographic damage over time in RA. 


\section{INTRODUCTION}

The identification of novel disease-related biomarkers is needed to improve early rheumatoid arthritis (RA) diagnosis, enable early initiation of appropriate treatment and to assess and predict disease severity. At present, no new biomarkers have yet matched or surpassed the clinical value of rheumatoid factor (RF) and anti-citrullinated protein autoantibodies (ACPA) [1]. These two autoantibodies are currently used as diagnostic tools, and their presence and titers are an integral part of the American College of Rheumatology (ACR)/ European League Against Rheumatism (EULAR) 2010 classification criteria [2].

RA patients were for many years subdivided into seropositive or seronegative RA according to their RF status. RFs are mostly measured as total RF (IgM, IgA and $\operatorname{IgG}$ ), with RF $\operatorname{IgM}$ representing the main isotype. However, in certain subgroups of patients, RF IgA and RF IgG are also present [3]. RF IgA is less sensitive than RF IgM but may be strongly linked to a more severe disease $[4,5]$. In general, RF exhibits limited specificity, as it is found in many other autoimmune diseases, infectious diseases, and in up to $15 \%$ of healthy elderly adults [3]. Depending on the population studied, RF has a specificity of $50-90 \%$ and a sensitivity of 60 $70 \%$ for the diagnosis of RA $[3,6,7]$.

Since the identification of ACPA, the distinction between ACPA positive and negative RA has been demonstrated to be even more discriminant, given the specificity of these antibodies as diagnostic markers, as well as their association with certain genetic markers (mainly in the MHC locus), environmental factors and more severe outcome measures [8-11]. The use of anticyclic citrullinated peptide (CCP) assays to detect ACPAs has enabled a prompt identification of individuals with RA and a better characterization of these patients, although clinicians still see some heterogeneity in anti-CCP positive RA patients. ACPA offers similar sensitivity, but higher specificity for RA than RF in early RA. In cohorts containing both established and early RA, the performance characteristics of RF and ACPA tests are comparable and the sensitivity of both assays is improved. [9, 12]. ACPA-positive RA is considered to be more severe than ACPA-negative RA because of its association with joint destruction. However, ACPA-positive RA is not more severe than ACPA-negative RA in terms of patient-reported outcomes such as pain, fatigue and work absenteeism [13].Therefore, seronegative RA patients may still develop severe disease outcomes, including erosive disease. Therefore, novel serological biomarkers are needed to close this serological gap and to better predict the prognosis of RA. 
Several new autoantibodies have been described in RA, including antibodies against carbamylated proteins (anti-CarP) that have been reported in the serum of RA patients with a sensitivity and specificity of $44 \%$ and $89 \%$, respectively [14-16]. Using a large cohort of sequential RA samples, it was demonstrated that anti-CarP antibodies predict joint damage, as measured by the total Sharp score (TSS) in both the ACPA-negative RA population and the overall RA population [14]. This association was replicated in several independent cohorts [1720]. Interestingly, a recent meta-analysis showed that the combination of anti-CarP antibodies, ACPAs, and RF had a very high specificity for the identification of patients with early RA [21]. Similar to RF and ACPA, these antibodies have also been observed years prior to the onset of clinical symptoms of RA, and they have been related to radiographic destruction at diagnosis and to worse radiographic progression in ACPA negative patients [22, 23].

Autoantibodies directed against the peptidyl-arginine deiminase (PAD) enzymes 2, 3 and 4 have also been detected in the serum of RA patients. Darrah et al. recently reported that antiPAD2 antibodies are associated with fewer swollen joints and less progression of joint damage [24]. In contrast, anti-PAD4 antibodies have been associated with disease severity and radiographic progression in RA [25-27]. As for ACPA, RF and anti-CarP antibodies, anti-PADs also precede the clinical onset of RA [28]. In addition, a subgroup of anti-PAD4 antibodies that cross-react with PAD3 [anti-PAD3/4 cross-reactive (XR) antibodies] was associated with more severe and progressive joint disease in both Caucasian and African American RA patients compared to the negative subgroup for these antibodies [29, 30]. In 2016, Seaman et al. reported the detection of anti-PAD3 antibodies for the first time by a chemiluminescent immunoassay (CIA) and a correlation with joint erosion score in a small cohort of RA patients $(n=39)$ [31]. However, additional studies are required to better assess the predictive value of anti-PAD3 on disease activity and the accrual of radiographic damage in RA.

The objective of this study was to assess the predictive value of anti-CarP and anti-PAD3 antibodies, alone or in combination with the other classical RA autoantibodies, on various RA outcomes including the 28-joint disease activity score (DAS28), the health assessment questionnaire (HAQ) score, and radiographic damage at baseline and over time, in a large cohort of established RA patients. 


\section{METHODS}

\section{Patients and sera}

All patients originated from the prospective "Swiss Clinical Quality Management (SCQM)" registry (http://scqm.ch) [32]. The registry longitudinally collects clinical information including disease activity and radiographic data from patients with RA, axial spondyloarthritis (axSpA) and psoriatic arthritis (PsA) (see online supplementary file for details).

\section{Immunoassays}

RFs IgM and IgA were determined by both QUANTA Lite ${ }^{\circledR}(\mathrm{QL})$ ELISAs (Inova Diagnostics). ACPA were determined by CCPlus Immunoscan ${ }^{\circledR}$ (anti-CCP2) IgG ELISA (Svar Life Science, Malmö, Sweden) and QL CCP3 IgG (Inova Diagnostics). Anti-CarP IgG antibodies were determined by a prototype ELISA using carbamylated fetal calf serum (Inova Diagnostics, RUO). Anti-PAD3 IgG antibodies were measured by a prototype CIA (Inova diagnostics, RUO) (see online supplementary file for details).

\section{Study Outcome definitions:}

Radiographic damage was centrally assessed within the SCQM registry by 2 evaluators, both of whom were unaware of clinical, sonographic and previous radiographic data, on digitalized radiographs of the hands and feet using the Ratingen (Rau) erosion score that evaluates 38 hand and foot joints $[33,34]$ (see online supplementary file for details).

The DAS28 and the HAQ scores were used to respectively assess disease activity and functional disability at baseline and over time $[35,36]$. Baseline was defined as the first visit with a Rheumatologist recorded in the registry. RA patients had a median of 4 radiographs [interquartile range (IQR) 2-6], a median of 15 HAQ assessments (IQR 9-24) and a median of 15 DAS28 assessments (IQR 9-24).

\section{Statistical analysis}

To examine the prognostic value of the various biomarkers on the evolution of functional disability (HAQ) and radiographic damage (Ratingen Score) over ten years, we used linear mixed-effects models including the biomarker, time and their interaction, and a random intercept and slope. Time was modelled with linear and quadratic terms. Multivariable analyses were corrected for age, sex, smoking status, disease duration, DAS28-ESR number of prior biologics and calendar year of biosampling. In order to investigate the potential changes of the 
biomarker levels over time, we explored for associations between the autoantibody titres and either the symptom duration or the age of patient, using Spearman's correlation analysis (see online supplementary file for details). 


\section{RESULTS}

The serological levels of each of the six biomarkers were determined in 851 patients with RA and 516 disease controls $(\operatorname{axSpA}=320$ and $\mathrm{PsA}=196)$. In the RA cohort, the various autoantibodies were positive in the following proportions: RF IgM 66.3\%, RF IgA 56.9\%, antiCCP2 73.2\%, anti-CCP3 63.8\%, anti-CarP 22.4\%, and anti-PAD3 10.7\% (Table 1). All six autoantibodies were also detected in a small proportion of disease controls, although at significantly lower rates than in RA patients.

Clinical performances for a diagnosis of RA for each biomarker are summarized in Table 1. Among the ACPA assays, anti-CCP2 showed the highest sensitivity (73.2\%), followed by antiCCP3 (63.8\%). The highest specificity was observed with anti-CCP3 (98.8\%) and RF IgM was more sensitive than RF IgA. When RF-IgM or anti-CCP2 were positive in the SpA control patients, the titers were generally much lower than in the auto-antibody positive RA patients with mean titers of 31.2 and 65.5 versus 134.9 and $928.3(\mathrm{p}<0.001)$, respectively (see supplementary Table S1).

Figure 1 demonstrates "UpSet" plots visualizing the diagnostic overlap of anti-CarP and antiPAD3 antibodies with RF IgM and anti-CCP3. As expected, the majority of RA patients were double positive for RF IgM and anti-CCP3 ( $\mathrm{n}=468 / 851,55.0 \%)$. Interestingly, there was a strong overlap between ACPA positivity and positivity to anti-PAD3 and anti-CarP. Nevertheless, $18.7 \%$ (17/91) of anti-PAD3-positive patients and 23.6\% (45/191) of anti-CarPpositive patients were anti-CCP3 negative (supp. Table S2). Anti-PAD3 and anti-CarP antibodies were present in $8.8 \%$, and $14.7 \%$ of patients who were seronegative for both RF IgM and anti-CCP3, respectively. Overlap results with $\mathrm{CCP} 2$ and $\mathrm{RF}$ IgA are shown in supplementary data (supp. Table S2 and Figure S1). In addition, anti-CCP3 titers were significantly higher in anti-PAD3 positive patients than in anti-PAD3 negative patients (median (IQR): $307.5(173,376)$ versus $255.6(94,359), \mathrm{p}=0.0115)$ (supp. Table S3).

The baseline characteristics of the RA patients according to autoantibody status are described in Table 2. Anti-CarP-positive patients were significantly less likely to be female than antiCarP-negative patients $(69.1 \%$ vs. $77.1 \%, p=0.024)$. Anti-PAD3-positive patients were significantly younger than anti-PAD3-negative patients (49.3 vs. 53.3 years, $p=0.005$ ), a difference that was not observed for anti-CarP. Anti-PAD3-positive patients were significantly less likely to be smokers than anti-PAD3-negative patients [45.3\% vs. 59.3\% $(p=0.01)]$. In 
contrast, there were no significant differences in the proportion of smokers with regards to positivity to RF-IgM, ACPA or anti-CarP.

Interestingly, anti-PAD3-positive patients had significantly higher baseline levels of the 28swollen joint count (SJC) and the DAS28-ESR score than anti-PAD3-negative patients: 7.1 vs. $4.3(p<0.0001)$ and 4.2 vs. $3.7(p=0.005)$, respectively. However, there were no differences with regards to anti-CarP positivity. The SJC was significantly higher in ACPA-positive patients compared with ACPA-negative-patients (4.9 vs. 4.1, $p=0.03$ ), although, this was not the case for the DAS28-ESR score as the TJC was somewhat higher in ACPA-negative patients. Regarding baseline levels of HAQ, there were no significant differences between autoantibodypositive and -negative patients for RF-IgM, anti-CCP3, anti-CarP or anti-PAD3 antibodies. Highly comparable results were obtained when the analyses were limited to RA patients with known positive ACR/EULAR criteria (results for anti-PAD3 can be found in supplementary table S3). Regarding the combinations of biomarkers, patients positive for five or six autoantibodies (RF IgM, RF IgA, anti-CCP3, anti-PAD3, anti-CarP +/- anti-CCP2) demonstrated a tendency towards higher baseline HAQ and DAS28-ESR scores, compared to patients with zero autoantibodies (Supplementary Table S4).

For all autoantibodies, the DAS28-ESR scores improved over the first few years following inclusion into the registry and then reached a steady plateau, with no significant differences between autoantibody positive and negative patients (data not shown). There were no significant differences in the evolution of the HAQ score over time between autoantibodypositive and -negative patients for any of the autoantibodies (data not shown). The different combinations of biomarkers were not associated with a greater progression of either the DAS28-ESR or HAQ scores over time compared to the total absence of biomarkers (Supplementary Table S4).

We further examined the association between autoantibodies individually and combined, and radiographic damage, at baseline and over time. In adjusted analyses, the presence of RF IgM, RF IgA and anti-CCP3 were significantly associated with higher baseline Ratingen scores ( $p=0.03, p<0.001$, and $p=<0.01$, respectively) (supp. Figure S2). Patients positive for antiPAD3 demonstrated significantly higher baseline Ratingen scores compared with anti-PAD3 negative patients (14.9 and 8.8 respectively) which was significant in both univariable and multivariable analyses ( $p<0.001$ and $p=0.02$ respectively) (Figure 2 ). 
The presence of multiple autoantibodies was generally associated with higher baseline Ratingen scores, particularly the combinations of RF IgM, anti-CCP3 and anti-PAD3, and similarly of RF IgM, RF IgA, anti-CCP3 and anti-PAD3, compared to those with absent autoantibodies (both $p<0.00001$ ) (Table 3). Regarding the combination of RF IgM, anti-CCP3 and anti-PAD3, the presence of all three autoantibodies predicted a significantly higher baseline Ratingen score than the presence of only two autoantibodies (14.4 vs. 10.4) $(p=0.04)$ (Figure 3A). Similarly, the presence of all four of the following autoantibodies: RF IgM, RF IgA, anti-CCP3 and antiPAD3, predicted a significantly higher baseline Ratingen score than the presence of only three autoantibodies (16.12 vs. 11.04) $(p=0.02)$ (Figure 3B).

Interestingly, in the anti-CCP3 negative subgroup of 308 patients (36.2\% of the RA population), baseline Ratingen scores were significantly higher in anti-PAD3 positive patients $(n=17)$ in both univariate analysis $(14.5$ vs. $6.6, p=0.01)$ and in multivariable adjusted analyses $(p=0.002)$ (data not shown). In the double seropositive (anti-CCP3 and RF IgM) subgroup of patients ( $\mathrm{n}=468,55.0 \%$ of the RA patients), anti-PAD3 positive patients ( $\mathrm{n}=64)$ demonstrated significantly higher baseline Ratingen scores compared with anti-PAD3-negative patients in univariable analyses ( 14.4 vs. $10.1, p=0.04)$, but not in multivariable analyses $(p=0.2)$ (data not shown). There were no significant differences with regards to anti-CarP, either in the whole RA population or in the seronegative subset (data not shown).

Regarding the impact of the individual antibodies on the evolution of radiographic damage over time, RF IgM and RF IgA were associated with significantly worse radiographic progression over 10 years ( $p=0.006$ and $p=0.04$ respectively) in adjusted analyses (Table 3 ). CCP3 IgG was associated with significantly worse radiographic progression over 10 years in univariable analyses $(p=0.049)$ and there was a trend in multivariable analyses $(p=0.0504)$. Interestingly CCP2 was not predictive of radiographic progression (data not shown). Radiographic progression over time between anti-PAD3 positive and negative patients (Figure 2), or between anti-CarP positive and negative patients (supp. Figure S2), was similar. In addition, we found that the presence of any two of the following autoantibodies: RF IgM, anti-CCP3 and antiPAD3, was associated with significantly greater radiographic progression over 10 years than if all three autoantibodies were absent $(p=0.02)$ (Figure 3A). The inclusion of anti-CarP, with RF IgM, anti-CCP3 and anti-PAD3, did not provide any additional benefit to the prediction of radiographic progression over time, over that of the other three autoantibodies (data not shown). 


\section{DISCUSSION}

We hereby describe the largest study of anti-PAD3 antibodies in RA patients and we demonstrate a significant association between anti-PAD3 positivity and markers of disease severity, such as the DAS28 and the Ratingen radiographic score. Interestingly, in the antiCCP3 seronegative group, higher baseline Ratingen scores were also observed in anti-PAD3 positive patients. In our cohort, the presence of anti-PAD3 alone did not predict greater radiographic progression over 10 years. This might be explained by the high percentage of patients with established and long-standing disease which might be different in an early RA cohort. However, in combination with multiple autoantibodies, anti-PAD3 antibodies were associated with further progression. Accordingly, we observed a dose effect on radiographic progression based on the number of positive biomarkers. Anti-PAD3 antibodies were recently detected with a novel CIA and were correlated with a joint erosion score in a small cohort of RA patients [31]. In our study, using the same assay, we confirmed the previous findings in a much larger cohort.

Previous studies have demonstrated the absence of synovial PAD3 expression in RA patients. In contrast, PAD2 and PAD4 proteins were detected in RA synovium in close association with tissue inflammation [37]. Competition experiments demonstrated that anti-PAD3 antibodies cross-react with PAD4 and a cross-reactive (XR) epitope between PAD3 and PAD4 enzymes was identified [29]. Interestingly, anti-PAD3/PAD4 positive RA patients have the most erosive joint disease when compared to anti-PAD negative patients or patients with anti-PAD4 antibodies. The potential cross-reactivity between anti-PAD3 antibodies and PAD2 and/or PAD4 antigens present in joints could support the biological relevance of anti-PAD3 antibodies as a biomarker of RA disease severity.

Recently, it was suggested that smoking history is not associated with anti-PAD positivity in RA patients, despite the strong association of anti-PAD4 antibodies and ACPA, as well as the accepted link between smoking and ACPA development [37-40]. Similarly, we observed that anti-PAD3-positive patients were significantly less likely to be smokers than anti-PAD3negative patients. Our results are supported by recent studies showing that smoking is not linked to the development of anti-PAD4 or anti-PAD3/4 cross reactive antibodies in RA patients [38, 41]. These results suggest that factors, other than cigarette smoking may drive the development of anti-PAD antibodies in patients with RA which remains a matter for future research. 
In contrast to our observations, Humphreys et al. demonstrated in an early arthritis cohort that the presence of anti-CarP antibodies were associated with increased disability defined by HAQ [42]. However, the effect size observed in their study did not meet some previously published 'minimum clinically important difference' for the HAQ [43]. In addition, we observed no significant differences at baseline for the DAS28 score with regards to anti-CarP positivity, although the DAS28 was numerically higher in the anti-CarP-positive group (3.86 vs. 3.68). Truchetet et al., described a small but significant difference in their early arthritis cohort, with a higher DAS28 in anti-CarP positive patients median (5.3 [95\% CI 5.2-5.5] vs. 5.0 [95\% CI 4.9-5.1]; $p=0.0004$ ) [18]. The DAS scores in their cohort were generally higher than in our study as it was an early arthritis population, which may explain these differences.

Regarding anti-CarP antibodies and radiographic damage, our findings differ from previous publications, which might be explained by the differences between the RA populations studied (early vs. long-standing disease) or by the methods of radiographic scoring (SHS vs. Ratingen score) $[14,17,18]$. In addition, it is important to note that previous studies adjusted for age, gender, inclusion period and CCP2, but not for smoking status or inflammation (CRP, DAS28), both of which have been reported to enhance carbamylation [44]. In addition, the discrepancies between the current study and previous studies in relation to anti-CarP antibodies and disease severity could be in part explained by the difference in sensitivity of $44 \%$ for anti-CarP antibodies in other RA cohorts, versus $22 \%$ in our cohort. Positivity for anti-CarP antibodies was demonstrated in $12.2 \%$ and $10.9 \%$ of PsA and axSpA patients respectively, with the majority of these Carp-positive SpA patients ( $88 \%$ and $72 \%$ respectively) being seronegative (for RF IgM and ACPA) (data not shown).Anti-Carp positivity was previously observed in active PsA patients and was recently described in 5/25 axSpA patients $[45,46]$. Our results suggest that the detection of anti-CarP antibodies alone at baseline in a long-standing RA population is not predictive for a more destructive disease course and might be restricted to early disease.

In contrast to anti-CCP3, we did not find a significant association between anti-CCP2 and either the baseline Ratingen scores or the progression of Ratingen scores over time. Similar discrepancies between CCP assays have been described in a previous study [47]. CCP tests were developed from different citrullinated antigens which may explain observed discrepancies. In comparison, van der Linden et al. observed no difference among anti-CCP2 and anti-CCP3 tests with regards to the ability to predict joint destruction [48]. Their analysis 
was performed on an early arthritis population, which may explain these discrepancies with our results in a cohort of RA patients with long-standing disease.

The strengths of this study are the large cohort size reflecting the general population with longstanding inflammatory joint disease, including 50\% of patients followed outside of academic institutions. In contrast to most other studies using anti-CCP2, we performed the majority of analyses with anti-CCP3 IgG, as these assays have previously demonstrated a superior clinical performance compared to anti-CCP2, when analysed individually as well as in combination with RF IgM [49]. In this present study, anti-CCP2 assay showed a lower specificity than antiCCP3 IgG assay ( $87.2 \%$ and $98.8 \%$, respectively). A potential explanation for the difference in specificity between the CCP2 and CCP3 assays could be the varying panels of citrullinated peptides of each test, and that some of the SpA disease controls have a higher degree of reactivity to the CCP2 peptide(s) as compared to the CCP3 peptide(s). However, whether the anti-CCP2 antibody response in disease controls is citrulline-specific has not been investigated. Indeed, "false-positive" CCP-reactivity has been described before in other disease control groups, where the reactivity to arginine-containing control peptides was analysed in parallel [50]. Consequently, it is potentially more difficult to demonstrate an additional benefit of a new autoantibody such as anti-PAD3 compared to anti-CCP3, than in comparison to anti-CCP2. The SCQM registry enabled us to analyse a large number of RA patients $(n=851)$ with a median of four radiographs (IQR 2-6) per patient and a follow-up of ten years. In contrast to most studies that utilized the Larsen or the Sharp/van der Heijde SHS scores, the Ratingen score was employed in our analyses. This radiographic scoring method was previously demonstrated to be reliable and precise, and suited to long-standing disease and to long-term observational studies in RA, especially in large-scale trials [33, 34]. Adjusted analyses were corrected for disease activity and the use of biological treatments, which was generally not the case in previous studies. Finally, the utilisation of an "active" disease control group of spondyloarthritis patients is more clinically relevant than a healthy control group to compare the performance of the different autoantibodies.

Nevertheless, a limitation of this study is that the biomarker sample was not systematically drawn at initiation of the first biologic agent. To minimize the impact of this limitation, we performed a statistical adjustment for the timing of the sample collection and the number of biologics utilized per patient. Serum levels of RF and ACPA seem to vary very little over time in the majority of RA patients, but it is currently unknown if the levels of other autoantibodies such as anti-PAD3 change significantly over time or are impacted by treatment. Nevertheless, 
we explored for a potential correlation between the level of each autoantibody and either the symptom duration or patient age and found no differences, except for RF IgA ( $r h o=0.12$, $p<0.001)$ and $\mathrm{RF} \operatorname{IgM}(r h o=0.09, p=0.01)$, suggesting that the other autoantibodies do not change significantly with aging. Another limitation of this study is that the overall radiographic damage progression over time was low due to a large proportion of patients treated with biologics, and therefore despite the fact that the observed differences were statistically different, they may not be "clinically" pertinent. Similarly, some of the differences between biomarkerpositive and biomarker-negative patients, while statistically significant, may not be clinically relevant. Finally, future studies are required to further evaluate the ideal cut-off values for these new biomarkers and to be vigilant with regards to the control population utilized.

In conclusion, this large study provides evidence for the role of anti-PAD3 antibodies as a potential biomarker of disease severity in RA patients. The combination of multiple autoantibodies, including anti-PAD3, RFs and ACPA, may be a useful tool to predict the severity of radiographic disease in RA.

\section{Conflict of interests}

M. Mahler and C. Bentow are employees of Inova Diagnostics; L. Martinez-Prat was previously employed at Inova Diagnostics. No share or stocks. All other authors declare no conflicts of interest.

\section{Funding statement}

This work was supported by the De Reuter Foundation.

\section{Acknowledgments and affiliations.}

The authors would like to thank patients and institutions who participated in SCQM (participating institutions are listed on www.scqm.ch/institutions). The SCQM is supported by sponsors (www.scqm.ch/sponsors). 


\section{References}

1. Bugatti S, Manzo A, Montecucco C, Caporali R. The Clinical Value of Autoantibodies in Rheumatoid Arthritis. Front Med (Lausanne). 2018;5:339.

2. Aletaha D, Neogi T, Silman AJ, Funovits J, Felson DT, Bingham CO, 3rd, et al. 2010

Rheumatoid arthritis classification criteria: an American College of Rheumatology/European League Against Rheumatism collaborative initiative. Arthritis Rheum. 2010;62(9):2569-2581.

3. Ingegnoli F, Castelli R, Gualtierotti R. Rheumatoid factors: clinical applications. Dis Markers. 2013;35(6):727-734.

4. Berglin E, Johansson T, Sundin U, Jidell E, Wadell G, Hallmans G, et al. Radiological outcome in rheumatoid arthritis is predicted by presence of antibodies against cyclic citrullinated peptide before and at disease onset, and by IgA-RF at disease onset. Ann Rheum Dis. 2006;65(4):453-458. 5. Jorgensen C, Legouffe MC, Bologna C, Brochier J, Sany J. IgA isotype rheumatoid factor in rheumatoid arthritis: clinical implications. Clin Exp Rheumatol. 1996;14(3):301-304.

6. Nishimura K, Sugiyama D, Kogata Y, Tsuji G, Nakazawa T, Kawano S, et al. Meta-analysis: diagnostic accuracy of anti-cyclic citrullinated peptide antibody and rheumatoid factor for rheumatoid arthritis. Ann Intern Med. 2007;146(11):797-808.

7. de Brito Rocha S, Baldo DC, Andrade LEC. Clinical and pathophysiologic relevance of autoantibodies in rheumatoid arthritis. Adv Rheumatol. 2019;59(1):2.

8. Vos I, Van Mol C, Trouw LA, Mahler M, Bakker JA, Van Offel J, et al. Anti-citrullinated protein antibodies in the diagnosis of rheumatoid arthritis (RA): diagnostic performance of automated antiCCP-2 and anti-CCP-3 antibodies assays. Clin Rheumatol. 2017;36(7):1487-1492.

9. Aggarwal R, Liao K, Nair R, Ringold S, Costenbader KH. Anti-citrullinated peptide antibody assays and their role in the diagnosis of rheumatoid arthritis. Arthritis Rheum. 2009;61(11):14721483.

10. Szodoray P, Szabo Z, Kapitany A, Gyetvai A, Lakos G, Szanto S, et al. Anti-citrullinated protein/peptide autoantibodies in association with genetic and environmental factors as indicators of disease outcome in rheumatoid arthritis. Autoimmun Rev. 2010;9(3):140-143.

11. Schellekens GA, de Jong BA, van den Hoogen FH, van de Putte LB, van Venrooij WJ. Citrulline is an essential constituent of antigenic determinants recognized by rheumatoid arthritis-specific autoantibodies. J Clin Invest. 1998;101(1):273-281.

12. Correia ML, Carvalho S, Fortuna J, Pereira MH. Comparison of three anti-CCP antibody tests and rheumatoid factor in RA and control patients. Clin Rev Allergy Immunol. 2008;34(1):21-25.

13. Boer AC, Boonen A, van der Helm van Mil AHM. Is Anti-Citrullinated Protein AntibodyPositive Rheumatoid Arthritis Still a More Severe Disease Than Anti-Citrullinated Protein AntibodyNegative Rheumatoid Arthritis? A Longitudinal Cohort Study in Rheumatoid Arthritis Patients Diagnosed From 2000 Onward. Arthritis Care Res (Hoboken). 2018;70(7):987-996.

14. Shi J, Knevel R, Suwannalai P, van der Linden MP, Janssen GM, van Veelen PA, et al. Autoantibodies recognizing carbamylated proteins are present in sera of patients with rheumatoid arthritis and predict joint damage. Proc Natl Acad Sci U S A. 2011;108(42):17372-17377.

15. Shi J, van de Stadt LA, Levarht EW, Huizinga TW, Toes RE, Trouw LA, et al. Anti-carbamylated protein antibodies are present in arthralgia patients and predict the development of rheumatoid arthritis. Arthritis Rheum. 2013;65(4):911-915.

16. Jiang X, Trouw LA, van Wesemael TJ, Shi J, Bengtsson C, Kallberg H, et al. Anti-CarP antibodies in two large cohorts of patients with rheumatoid arthritis and their relationship to genetic risk factors, cigarette smoking and other autoantibodies. Ann Rheum Dis. 2014;73(10):1761-1768. 17. Ajeganova S, van Steenbergen HW, Verheul MK, Forslind K, Hafstrom I, Toes RE, et al. The association between anti-carbamylated protein (anti-CarP) antibodies and radiographic progression in early rheumatoid arthritis: a study exploring replication and the added value to ACPA and rheumatoid factor. Ann Rheum Dis. 2017;76(1):112-118.

18. Truchetet ME, Dublanc S, Barnetche T, Vittecoq O, Mariette X, Richez C, et al. Association of the Presence of Anti-Carbamylated Protein Antibodies in Early Arthritis With a Poorer Clinical and 
Radiologic Outcome: Data From the French ESPOIR Cohort. Arthritis Rheumatol. 2017;69(12):22922302.

19. Yee A, Webb T, Seaman A, Infantino M, Meacci F, Manfredi M, et al. Anti-CarP antibodies as promising marker to measure joint damage and disease activity in patients with rheumatoid arthritis. Immunol Res. 2015;61(1-2):24-30.

20. Elsayed SA, Esmail MA, Ali RM, Mohafez OM. Diagnostic and prognostic value of anti-CarP antibodies in a sample of Egyptian rheumatoid arthritis patients. Clin Rheumatol. 2019.

21. Verheul MK, Bohringer S, van Delft MAM, Jones JD, Rigby WFC, Gan RW, et al. Triple Positivity for Anti-Citrullinated Protein Autoantibodies, Rheumatoid Factor, and Anti-Carbamylated Protein Antibodies Conferring High Specificity for Rheumatoid Arthritis: Implications for Very Early Identification of At-Risk Individuals. Arthritis Rheumatol. 2018;70(11):1721-1731.

22. Brink M, Verheul MK, Ronnelid J, Berglin E, Holmdahl R, Toes RE, et al. Anti-carbamylated protein antibodies in the pre-symptomatic phase of rheumatoid arthritis, their relationship with multiple anti-citrulline peptide antibodies and association with radiological damage. Arthritis Res Ther. 2015;17:25.

23. Shi J, van de Stadt LA, Levarht EW, Huizinga TW, Hamann D, van Schaardenburg D, et al. Anticarbamylated protein (anti-CarP) antibodies precede the onset of rheumatoid arthritis. Ann Rheum Dis. 2014;73(4):780-783.

24. Darrah E, Giles JT, Davis RL, Naik P, Wang H, Konig MF, et al. Autoantibodies to Peptidylarginine Deiminase 2 Are Associated With Less Severe Disease in Rheumatoid Arthritis. Front Immunol. 2018;9:2696.

25. Halvorsen EH, Pollmann S, Gilboe IM, van der Heijde D, Landewe R, Odegard S, et al. Serum IgG antibodies to peptidylarginine deiminase 4 in rheumatoid arthritis and associations with disease severity. Ann Rheum Dis. 2008;67(3):414-417.

26. Harris ML, Darrah E, Lam GK, Bartlett SJ, Giles JT, Grant AV, et al. Association of autoimmunity to peptidyl arginine deiminase type 4 with genotype and disease severity in rheumatoid arthritis. Arthritis Rheum. 2008;58(7):1958-1967.

27. Halvorsen EH, Haavardsholm EA, Pollmann S, Boonen A, van der Heijde D, Kvien TK, et al. Serum IgG antibodies to peptidylarginine deiminase 4 predict radiographic progression in patients with rheumatoid arthritis treated with tumour necrosis factor-alpha blocking agents. Ann Rheum Dis. 2009;68(2):249-252.

28. Kolfenbach JR, Deane KD, Derber LA, O'Donnell Cl, Gilliland WR, Edison JD, et al. Autoimmunity to peptidyl arginine deiminase type 4 precedes clinical onset of rheumatoid arthritis. Arthritis Rheum. 2010;62(9):2633-2639.

29. Darrah E, Giles JT, Ols ML, Bull HG, Andrade F, Rosen A. Erosive rheumatoid arthritis is associated with antibodies that activate PAD4 by increasing calcium sensitivity. Sci Trans/ Med. 2013;5(186):186ra165.

30. Navarro-Millan I, Darrah E, Westfall AO, Mikuls TR, Reynolds RJ, Danila MI, et al. Association of anti-peptidyl arginine deiminase antibodies with radiographic severity of rheumatoid arthritis in African Americans. Arthritis Res Ther. 2016;18(1):241.

31. Seaman A, Darrah E, Infantino M, Meacci F, Manfredi M, Benucci M, et al. Anti-peptidylarginine deaminase 3 (PAD3) antibodies as a promising marker to measure joint damage in patients with rheumatoid arthritis. Autoimmun Rev. 2016;15(7):776-780.

32. Ciubotariu E, Gabay C, Finckh A, Physicians of the Swiss Clinical Quality Management Program for Rheumatoid A. Joint damage progression in patients with rheumatoid arthritis in clinical remission: do biologics perform better than synthetic antirheumatic drugs? J Rheumatol. 2014;41(8):1576-1582.

33. Rau R, Wassenberg S, Herborn G, Stucki G, Gebler A. A new method of scoring radiographic change in rheumatoid arthritis. J Rheumatol. 1998;25(11):2094-2107.

34. Tanaka E, Yamanaka H, Matsuda Y, Urano W, Nakajima H, Taniguchi A, et al. Comparison of the Rau method and the Larsen method in the evaluation of radiographic progression in early rheumatoid arthritis. J Rheumatol. 2002;29(4):682-687. 
35. van Riel PL, Renskers L. The Disease Activity Score (DAS) and the Disease Activity Score using 28 joint counts (DAS28) in the management of rheumatoid arthritis. Clin Exp Rheumatol. 2016;34(5 Suppl 101):S40-S44.

36. Guillemin F, Briancon S, Pourel J. Validity and discriminant ability of the HAQ Functional Index in early rheumatoid arthritis. Disabil Rehabil. 1992;14(2):71-77.

37. Foulquier C, Sebbag M, Clavel C, Chapuy-Regaud S, Al Badine R, Mechin MC, et al. Peptidyl arginine deiminase type 2 (PAD-2) and PAD- 4 but not PAD-1, PAD-3, and PAD- 6 are expressed in rheumatoid arthritis synovium in close association with tissue inflammation. Arthritis Rheum. 2007;56(11):3541-3553.

38. Cappelli LC, Konig MF, Gelber AC, Bingham CO, 3rd, Darrah E. Smoking is not linked to the development of anti-peptidylarginine deiminase 4 autoantibodies in rheumatoid arthritis. Arthritis Res Ther. 2018;20(1):59.

39. Kokkonen H, Brink M, Hansson M, Lassen E, Mathsson-Alm L, Holmdahl R, et al. Associations of antibodies against citrullinated peptides with human leukocyte antigen-shared epitope and smoking prior to the development of rheumatoid arthritis. Arthritis Res Ther. 2015;17:125.

40. Roos K, Martinsson K, Ziegelasch M, Sommarin Y, Svard A, Skogh T, et al. Circulating secretory IgA antibodies against cyclic citrullinated peptides in early rheumatoid arthritis associate with inflammatory activity and smoking. Arthritis Res Ther. 2016;18(1):119.

41. Darrah E, Yu F, Cappelli LC, Rosen A, O'Dell JR, Mikuls TR. Association of Baseline Peptidylarginine Deiminase 4 Autoantibodies With Favorable Response to Treatment Escalation in Rheumatoid Arthritis. Arthritis Rheumatol. 2019;71(5):696-702.

42. Humphreys J, Verheul M, Barton A, Fu B, Toes R, Symmons D, et al. Association of anticarbamylated protein antibodies with long-term disability and increased disease activity in patients with early inflammatory arthritis: results from the Norfolk Arthritis Register. Lancet. 2015;385 Suppl 1:S44.

43. Kosinski M, Zhao SZ, Dedhiya S, Osterhaus JT, Ware JE, Jr. Determining minimally important changes in generic and disease-specific health-related quality of life questionnaires in clinical trials of rheumatoid arthritis. Arthritis Rheum. 2000;43(7):1478-1487.

44. Wang Z, Nicholls SJ, Rodriguez ER, Kummu O, Horkko S, Barnard J, et al. Protein carbamylation links inflammation, smoking, uremia and atherogenesis. Nat Med. 2007;13(10):11761184.

45. Chimenti MS, Triggianese P, Nuccetelli M, Terracciano C, Crisanti A, Guarino MD, et al. Autoreactions, autoimmunity and psoriatic arthritis. Autoimmun Rev. 2015;14(12):1142-1146.

46. Sidiras P, Spruyt D, Gangji V, Imbault V, Sokolova T, Durez P, et al. Antibodies against carbamylated proteins: prevalence and associated disease characteristics in Belgian patients with rheumatoid arthritis or other rheumatic diseases. Scand J Rheumatol. 2020:1-6.

47. Innala L, Kokkonen H, Eriksson C, Jidell E, Berglin E, Dahlqvst SR. Antibodies against mutated citrullinated vimentin are a better predictor of disease activity at 24 months in early rheumatoid arthritis than antibodies against cyclic citrullinated peptides. J Rheumatol. 2008;35(6):1002-1008. 48. van der Linden MP, van der Woude D, Ioan-Facsinay A, Levarht EW, Stoeken-Rijsbergen G, Huizinga TW, et al. Value of anti-modified citrullinated vimentin and third-generation anti-cyclic citrullinated peptide compared with second-generation anti-cyclic citrullinated peptide and rheumatoid factor in predicting disease outcome in undifferentiated arthritis and rheumatoid arthritis. Arthritis Rheum. 2009;60(8):2232-2241.

49. Martinez-Prat L, Nissen MJ, Lamacchia C, Bentow C, Cesana L, Roux-Lombard P, et al. Comparison of Serological Biomarkers in Rheumatoid Arthritis and Their Combination to Improve Diagnostic Performance. Front Immunol. 2018;9:1113.

50. Vannini A, Cheung K, Fusconi M, Stammen-Vogelzangs J, Drenth JP, Dall'Aglio AC, et al. Anticyclic citrullinated peptide positivity in non-rheumatoid arthritis disease samples: citrulline-

dependent or not? Ann Rheum Dis. 2007;66(4):511-516. 
Figure 1: Autoantibody positivity in rheumatoid arthritis (RA) patients. These UpSet plots show the diagnostic overlap of (A) three autoantibodies (RF IgM, anti-CCP3 and anti-PAD3), (B) four autoantibodies (RF IgM, anti-CCP3, anti-PAD3 and anti-CarP) or (C) five autoantibodies (RF IgA, RF IgM, anti-CCP3, anti-PAD3 and anti-Carp), in RA patients. The number of RA patients positive for the different combinations are indicated in the top bar chart. The presence of each autoantibody is indicated with filled circles below the bar chart. Bars on the left depict the number of positive samples for each autoantibody.
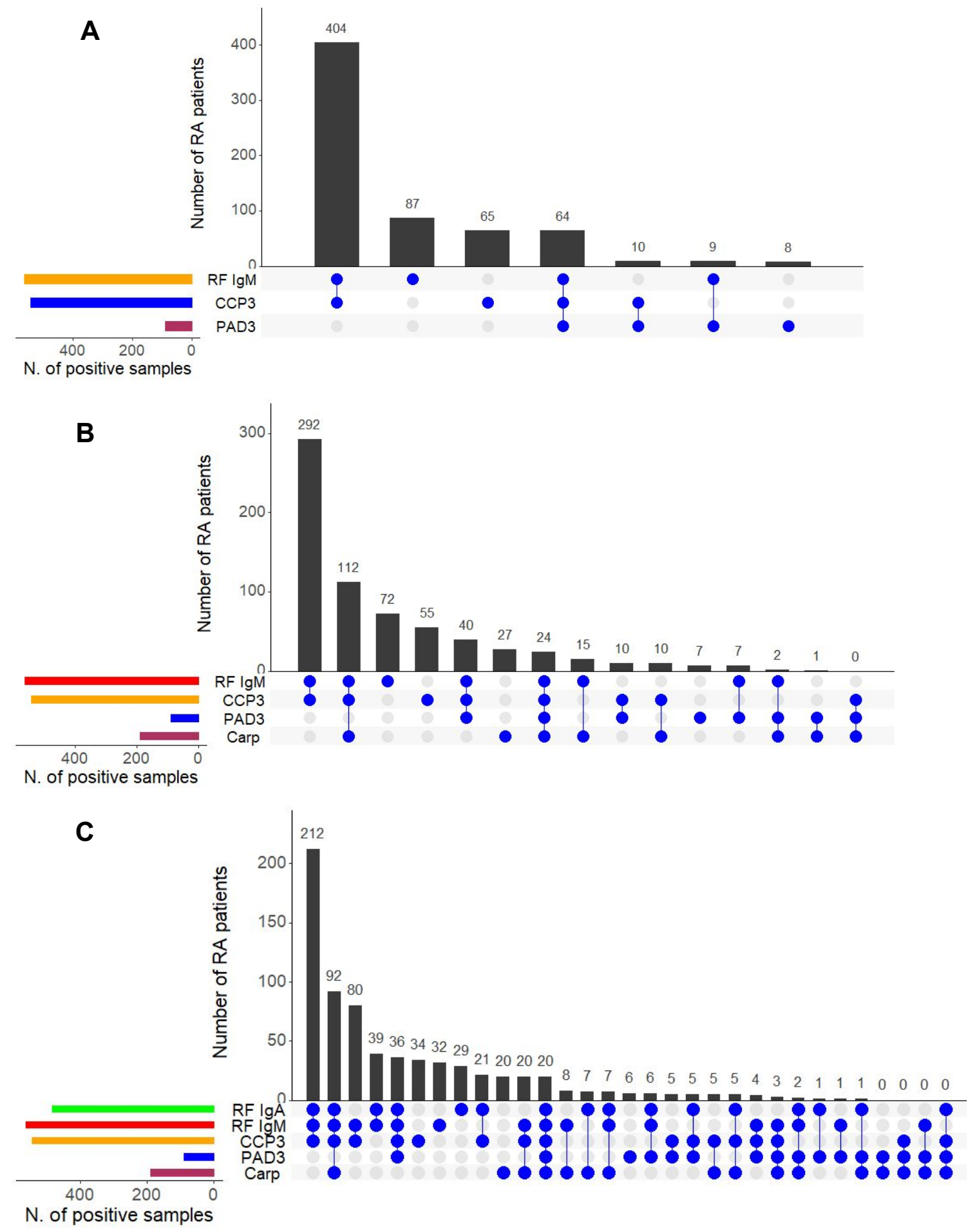
Figure 2: Association of anti-PAD3 antibody with radiographic damage at baseline and over time in rheumatoid arthritis (RA) population. The analysis of Ratingen scores (percentage of the total score) was based on the presence or absence of the anti-PAD3 antibody. Number of patients per subgroup: anti-PAD3 negative: 760; anti-PAD3 positive: 91.

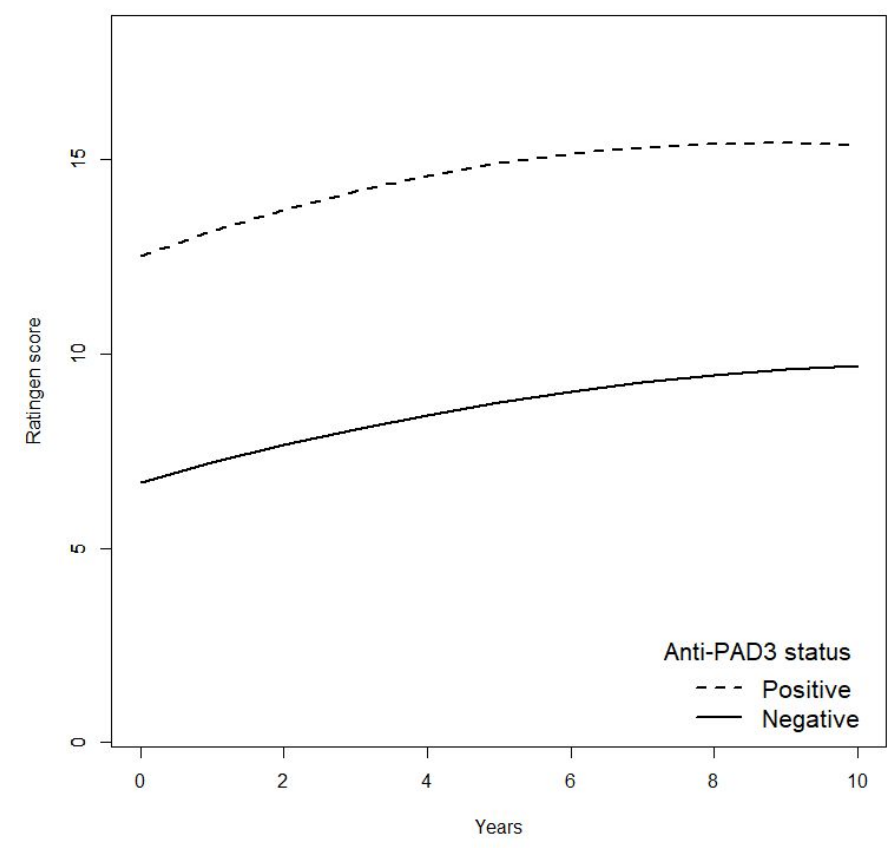


Figure 3: Association between the number of autoantibodies and radiographic damage at baseline and over time in rheumatoid arthritis (RA) population. The analysis of Ratingen scores (percentage of the total score) were based on the number of autoantibodies present (A: RF IgM, CCP3 and anti-PAD3; B: RF IgM, RF IgA, CCP3 and anti-PAD3). The number of patients per biomarker subgroup appears to the right of each panel.

A

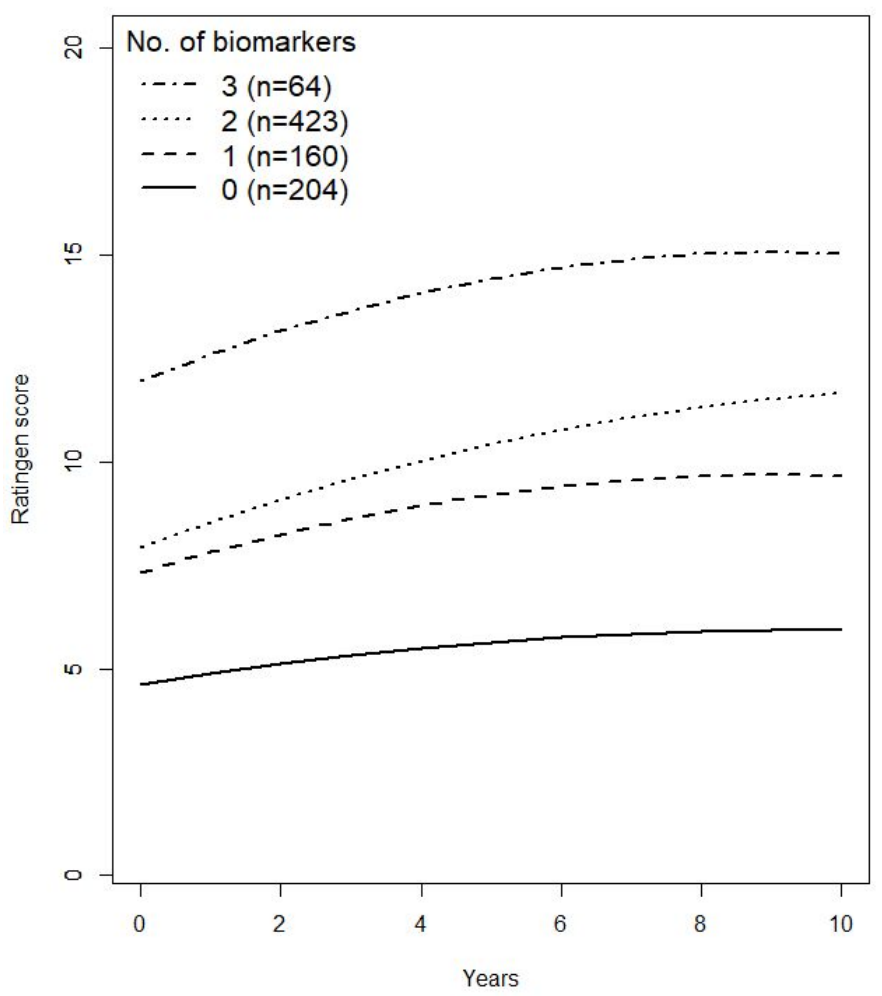

B

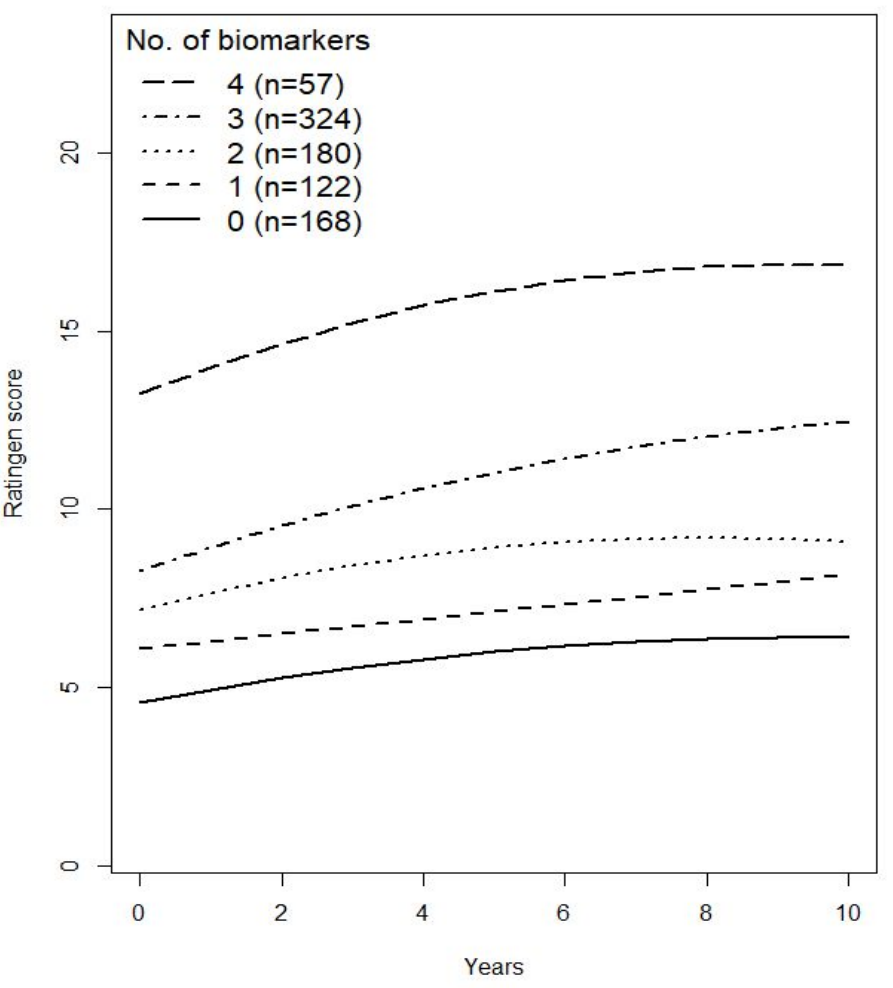


Table 1: Autoantibody frequency in patients with RA, PsA and axSpA, and the sensitivities, specificities, PPV and NPV for a diagnosis of RA as defined by the treating rheumatologist. Sens=sensitivity, $\mathrm{Spec}=$ specificity, $\mathrm{PPV}=$ positive predictive value, NPV=negative predictive value, IU =International Units, RLU $=$ relative light unit. ${ }^{*} \mathrm{p}<0.01$ compared to the RA population, ${ }^{* *} \mathrm{p}<0.001$ compared to the RA population; $¥ 2$ missing values ( 1 in axSpA and 1 in RA).

\begin{tabular}{|c|c|c|c|c|c|c|c|c|c|}
\hline \multirow[t]{3}{*}{ Biomarker } & \multirow{3}{*}{$\begin{array}{l}\text { Cut-off } \\
\text { (units) }\end{array}$} & \multicolumn{4}{|c|}{ Percentage of positive samples } & \multirow{3}{*}{$\begin{array}{c}\text { Sens } \\
(\%)\end{array}$} & \multirow{3}{*}{$\begin{array}{c}\text { Spec } \\
(\%)\end{array}$} & \multirow{3}{*}{$\begin{array}{l}\text { PPV } \\
(\%)\end{array}$} & \multirow{3}{*}{$\begin{array}{l}\text { NPV } \\
\text { (\%) }\end{array}$} \\
\hline & & Overall & RA & PsA & axSpA & & & & \\
\hline & & $\mathrm{N}=1367$ & $\mathrm{~N}=851$ & $\mathrm{~N}=196$ & $\mathrm{~N}=320$ & & & & \\
\hline RF IgM & $6 \mathrm{IU}$ & 45.9 & 66.3 & $12.2^{* *}$ & $13.1^{* *}$ & 66.3 & 87.6 & 89.8 & 61.2 \\
\hline$R F \lg A$ & $6 \mathrm{IU}$ & 43.9 & 56.9 & $21.4^{* *}$ & $23.1^{* *}$ & 56.9 & 77.5 & 80.7 & 52.2 \\
\hline Anti-CCP2 $^{\ddagger}$ & $25 \mathrm{IU}$ & 50.4 & 73.2 & $12.2^{* *}$ & $13.2^{* *}$ & 73.2 & 87.2 & 90.4 & 66.3 \\
\hline Anti-CCP3 & $20 \mathrm{IU}$ & 40.2 & 63.8 & $0.5^{\star \star}$ & $1.6^{* *}$ & 63.8 & 98.8 & 98.9 & 62.3 \\
\hline Anti-CarP & $20 \mathrm{IU}$ & 18.3 & 22.4 & $12.2^{*}$ & $10.9^{* *}$ & 22.4 & 88.6 & 76.4 & 59.1 \\
\hline Anti- PAD3 & 8300 RLU & 7.2 & 10.7 & $1.5^{\star \star}$ & $1.3^{\star \star}$ & 10.7 & 98.6 & 92.9 & 40.1 \\
\hline
\end{tabular}


Table 2: Baseline characteristics of rheumatoid arthritis (RA) patients according to autoantibody status. Abbreviations: Disease dur. $=$ disease duration; yrs = years; $\mathrm{SD}=$ standard deviation; IQR = interquartile range; $\mathrm{TJC}=$ tender joint count; $\mathrm{SJC}=$ swollen joint count; DAS = disease activity score; $\mathrm{CRP}=\mathrm{C}$ reactive protein; ESR = erythrocyte sedimentation rate; $\mathrm{HAQ}=$ health assessment questionnaire; anti-CarP = anti-carbamylated proteins antibodies; anti-PAD3

$=$ anti-peptidyl arginine deiminase type- 3 antibodies; $\mathrm{RF}=$ rheumatoid factor; ACPA = anti-citrullinated protein antibodies. RF and ACPA status were represented by RF IgM and anti-CCP3 assays, respectively. ${ }^{\mathrm{t}}=\operatorname{trend}(\mathrm{p}<0.10),{ }^{*} \mathrm{p}<0.05,{ }^{* *} \mathrm{p}<0.01,{ }^{* * *} \mathrm{p}<0.001$.

\begin{tabular}{|c|c|c|c|c|c|c|c|c|c|c|}
\hline Baseline characteristics & $\mathbf{N}$ & $\begin{array}{c}\mathrm{RA} \\
(\mathrm{N}=851)\end{array}$ & $\begin{array}{l}R F \lg M+ \\
(\mathrm{N}=533)\end{array}$ & $\begin{array}{l}\text { RF IgM- } \\
(\mathrm{N}=264)\end{array}$ & $\begin{array}{l}\text { ACPA+ } \\
(\mathrm{N}=543)\end{array}$ & $\begin{array}{l}\text { ACPA- } \\
(\mathrm{N}=\mathbf{3 0 8})\end{array}$ & $\begin{array}{c}\text { Anti-CarP+ } \\
(\mathrm{N}=191)\end{array}$ & $\begin{array}{c}\text { Anti-CarP- } \\
(\mathrm{N}=660)\end{array}$ & $\begin{array}{c}\text { Anti-PAD3+ } \\
(\mathrm{N}=91)\end{array}$ & $\begin{array}{c}\text { Anti-PAD3- } \\
(\mathrm{N}=760)\end{array}$ \\
\hline Age (years) (mean, SD) & 851 & $52.9(13.0)$ & $52.2(12.9)^{*}$ & $54.2(12.9)$ & $52.6(12.6)$ & $53.4(13.6)$ & $53.2(13.2)$ & $52.8(12.9)$ & $49.3(12.5)^{\star \star}$ & $53.3(12.9)$ \\
\hline Female gender (\%) & 851 & 75.3 & 75.5 & 74.9 & 75.0 & 76.0 & $69.1^{*}$ & 77.1 & 76.9 & 75.1 \\
\hline Weight (kg) & 820 & 70.8 & 70.3 & 71.1 & 70.4 & 71.4 & 72.0 & 70.4 & 70.7 & 70.8 \\
\hline Ever Smoker (\%) & 809 & 57.8 & 58.7 & 56.1 & 59.2 & 55.5 & 58.1 & 57.8 & $45.3^{*}$ & 59.3 \\
\hline Alcohol consumption (\%) & 813 & 72.9 & 73.1 & 72.6 & 72.6 & 73.6 & 75.0 & 72.3 & 70.4 & 73.2 \\
\hline Higher education (\%) & 851 & 72.2 & 72.2 & 72.1 & 70.7 & 74.7 & 72.8 & 72.0 & 69.2 & 72.5 \\
\hline Disease dur. (yrs) (med, IQR) & 819 & $4.55(1.6,10.9)$ & $4.80(1.6,11.2)$ & $3.90(1.5,8.8)$ & $4.76(1.7,11.2)$ & $4.28(1.5,9.4)$ & $4.50(1.6,11.8)$ & $4.55(1.6,10.6)$ & $6.62(2.3,12.9)^{*}$ & $4.27(1.5,10.4)$ \\
\hline Raised CRP or ESR (\%) & 851 & 51.5 & $54.4^{*}$ & 45.6 & $55.1^{\star \star}$ & 45.1 & 56.5 & 50.0 & $60.4^{\mathrm{t}}$ & 50.4 \\
\hline Followed in a univ. hospital (\%) & 851 & 50.4 & 52.1 & 47.0 & $52.7^{\mathrm{t}}$ & 46.4 & 49.2 & 50.8 & $68.1^{\star \star *}$ & 48.3 \\
\hline ACR-EULAR criteria positive (\%) & 851 & 86.1 & $94.9^{\star \star *}$ & 69.0 & $96.1^{1 * *}$ & 68.5 & 91.1* & 84.7 & $95.6^{\star *}$ & 85.0 \\
\hline Anti-CCP3 positive (\%) & 851 & 63.8 & $83.0^{\star \star *}$ & 26.1 & 100 & 0 & $76.4^{\star \star *}$ & 60.2 & $81.3^{\star \star *}$ & 61.7 \\
\hline RF positive (\%) & 851 & 66.3 & 100 & 0 & $86.2^{\star \star \star}$ & 31.2 & $80.1^{* \star *}$ & 62.3 & $80.2^{\star \star}$ & 64.6 \\
\hline Physician global (mean, SD) & 839 & $3.3(2.3)$ & $3.3(2.4)$ & $3.1(2.1)$ & $3.4(2.4)$ & $3.1(2.2)$ & $3.4(2.4)$ & $3.2(2.3)$ & $3.6(2.6)$ & $3.2(2.3)$ \\
\hline TJC (/28) (mean, SD) & 851 & $5.2(6.1)$ & $5.2(6.2)$ & $5.3(5.7)$ & $5.1(5.9)$ & $5.5(6.3)$ & $5.5(6.6)$ & $5.1(5.9)$ & $6.8(7.7)^{\star \star}$ & $5.0(5.8)$ \\
\hline SJC (/28) (mean, SD) & 851 & $4.6(5.3)$ & $4.8(5.5)$ & $4.2(5.0)$ & $4.9(5.4)^{*}$ & $4.1(5.3)$ & $4.9(5.7)$ & $4.5(5.2)$ & $7.1(7.1)^{\star \star \star}$ & $4.3(5.0)$ \\
\hline DAS28-ESR (mean, SD) & 849 & $3.7(1.6)$ & $3.8(1.6)$ & $3.6(1.5)$ & $3.8(1.6)$ & $3.6(1.6)$ & $3.9(1.5)$ & $3.7(1.6)$ & $4.2(1.8)^{\star \star}$ & $3.7(1.5)$ \\
\hline HAQ (med, IQR) & 831 & $0.75(0.25,1.375)$ & $0.75(0.25,1.375)$ & 0.875 & $0.75(0.25,1.375)$ & $0.875(0.25,1.375)$ & $0.625(0.25,1.25)$ & $0.875(0.25,1.375)$ & $0.875(0.375,1.375)$ & $0.75(0.25,1.375$ \\
\hline SF-12 mental (mean, SD) & 797 & $45.7(12.2)$ & $46.0(12.1)$ & $45.2(12.2)$ & $45.9(12.1)$ & $45.5(12.3)$ & $46.4(12.2)$ & $45.5(12.2)$ & $47.1(12.0)$ & $45.6(12.2)$ \\
\hline SF-12 physical (mean, SD) & 797 & $38.5(10.5)$ & $38.5(10.6)$ & $38.7(10.3)$ & $38.6(10.6)$ & $38.5(10.4)$ & $38.5(10.3)$ & $38.6(10.6)$ & $37.9(10.4)$ & $38.6(10.5)$ \\
\hline Prior biologic use (\%) & 851 & 56.4 & 57.6 & 54.0 & 58.2 & 53.2 & 53.4 & 57.2 & 56.0 & 56.4 \\
\hline
\end{tabular}


Table 3: Radiographic damage at baseline and over time in the RA population with regards to the individual autoantibodies and their combinations. The P-value represents the comparison of autoantibody positive versus autoantibody negative patients for the individual autoantibodies and compared to zero autoantibodies for the autoantibody combinations. No. $=$ number, AAbs $=$ autoantibodies, $\mathrm{SE}=$ standard error.

\begin{tabular}{|c|c|c|c|c|c|c|c|c|}
\hline & \multirow{2}{*}{$\begin{array}{l}\text { No. of } \\
\text { AAbs }\end{array}$} & \multirow{2}{*}{$\begin{array}{l}\text { No. of } \\
\text { patients }\end{array}$} & \multicolumn{3}{|c|}{ Baseline Ratingen score } & \multicolumn{3}{|c|}{ Increase in Ratingen units / year } \\
\hline & & & $\begin{array}{l}\text { Univariable } \\
\mathrm{N}=\mathbf{8 5 1}\end{array}$ & & $\begin{array}{l}\text { Multivariable } \\
\mathrm{N}=779\end{array}$ & $\begin{array}{l}\text { Univariable } \\
\mathrm{N}=\mathbf{8 5 1}\end{array}$ & & $\begin{array}{l}\text { Multivariable } \\
\mathrm{N}=779\end{array}$ \\
\hline & & & Score (SE) & $p$-value & $\mathrm{p}$-value & Score (SE) & p-value & $p$-value \\
\hline \multicolumn{9}{|l|}{ Individual AAb } \\
\hline RF IgM & 1 & 564 & $10.46(1.08)$ & 0.004 & 0.03 & $0.20(0.08)$ & 0.01 & 0.006 \\
\hline RF IgA & 1 & 484 & $11.17(1.03)$ & $<0.00001$ & 0.0005 & $0.12(0.07)$ & 0.11 & 0.04 \\
\hline Anti-CCP3 & 1 & 543 & $10.73(1.07)$ & 0.0004 & 0.004 & $0.16(0.08)$ & 0.049 & 0.05 \\
\hline Anti-PAD3 & 1 & 91 & $14.90(1.63)$ & 0.0002 & 0.018 & $0.01(0.11)$ & 0.90 & 0.95 \\
\hline \multicolumn{9}{|l|}{ AAb combinations } \\
\hline \multirow{4}{*}{$\begin{array}{l}\text { RF IgM, anti-CCP3 } \\
\text { and anti-PAD3 }\end{array}$} & 0 & 204 & $5.63(1.06)$ & - & - & $0.13(0.09)$ & - & - \\
\hline & 1 & 160 & $9.20(1.57)$ & 0.02 & 0.047 & $0.23(0.12)$ & 0.39 & 0.63 \\
\hline & 2 & 423 & $10.44(1.28)$ & 0.0002 & 0.001 & $0.37(0.10)$ & 0.02 & 0.02 \\
\hline & 3 & 64 & $14.42(2.11)$ & 0.00004 & 0.002 & $0.31(0.15)$ & 0.24 & 0.25 \\
\hline \multirow{5}{*}{$\begin{array}{l}\text { RF IgM, RF IgA, } \\
\text { anti-CCP3 and anti- } \\
\text { PAD3 }\end{array}$} & 0 & 168 & $6.01(1.16)$ & - & - & $0.19(0.10)$ & - & - \\
\hline & 1 & 122 & $7.13(1.78)$ & 0.53 & 0.77 & $0.21(0.14)$ & 0.87 & 0.74 \\
\hline & 2 & 180 & $8.92(1.59)$ & 0.07 & 0.21 & $0.19(0.12)$ & 0.97 & 0.69 \\
\hline & 3 & 324 & $11.04(1.42)$ & 0.0004 & 0.002 & $0.42(0.11)$ & 0.04 & 0.04 \\
\hline & 4 & 57 & $16.12(2.26)$ & $<0.00001$ & 0.002 & $0.36(0.16)$ & 0.29 & 0.32 \\
\hline
\end{tabular}

